\title{
IUPAC Commission on the Nomenclature of Organic Chemistry (CNOC) and IUPAC-IUB Commission on Biochemical Nomenclature (CBN)
}

\section{Nomenclature of Cyclitols}

\author{
Recommendations, $1973^{1}$
}

\section{INTRODUCTION}

\section{Origin}

These Recommendations are issued jointly by the Commission on the Nomenclature of Organic Chemistry $^{2}$ of IUPAC and by the IUPAC.IUB Commission on Biochemical Nomenclature ${ }^{3}$. They are the outgrowth of Tentative Rules [1] issued in 1968 on the basis of a report by a Joint Cyclitol Nomenclature Sub-Committee ${ }^{4}$.

\section{Scope of Cyclitol Nomenclature}

Cyclitols are cycloalkanes containing one hydroxyl group on each of three or more ring atoms ${ }^{5}$. These compounds, and others closely related to them, possess features of relative and absolute configuration that are characteristic of their class and have been extensively studied; but these features are not clearly displayed by general methods of stereochemical nomenclature, so that special methods of specifying their configuration are justified and have long been used. In other than stereochemical respects, their nomenclature should follow the general rules of organic chemistry.

${ }^{1}$ These Recommendations shall be known as the IUPAC-IUB 1973 Recommendations for Cyclitol. Comments on and suggestions for future revisions of these Recommendations should be sent to Professor N. Lozac'h, Ecole Nationale Supérieure de Chimie, 5 Avenue d'Edimbourg; F-14032 Caen-Cedex, France, or to Professor O. Hoffmann-Ostenhiof, Institut für Allgemeine Biochemie der Univerrsität Wien, Währingerstraße 38, A-1090 Wien, Austrła, or to any present member of the commissions named below.

${ }^{2}$ Titular members: N. Lozac'h (chalirman), S. P. Klesney (secretary), K. Bláha, L. C. Cross, H, Grünewald, W. Klyne, K. L. Loening, and J. Rigaudy, Associate. members: K. Hirayama, $S$. Veibel, and F. Vögtle.

Members: O. Hoffmann-Ostenhof (chairman), W. E. Cohn (secretary), A.E. Braunstein, B.L. Horecker, W. B.Jakoby, P. Karlson, B. Keil, W, Klyne, C. Liébecq, and E. C. Webb.

${ }^{4}$ S. J. Angyal (chairman), L. Anderson, R. S. Cahn, R. M. C. Dawson, Q. Hoffmann-Ostenhof, W. Klyne, and T, Posternak.

${ }^{3}$ Cycloalkanes containing fewer than three hydroxyl groups are better named by the more general methods of organic chemical nomenclature.
The sequence-rule $(R, S)$ system $[2,3]$ may be used when it is desired to relate a cyclitol to the general stereochemical natation and for describing the configuration of chiral groups, such as benzylidene, which may be present as substituents; but the procedures described below are recommended for cyclitol chemistry as such because of the complexity of the sequencerule procedure in this field.

The nomenclature described below is most useful for cycloalkanes containing only one kind of substituent, and especially for cyclitols and their esters and ethers, but it may also be applied to their derivatives in which one or more hydroxyl groups have been replaced by other groups.

\section{Evolution of Cyclitol Nomenclature}

The typical stereochemical feature of cyclitols is exemplified by formula $A$, usually drawn more simply as B or C, in which the ring is considered as being planar and nearly perpendicular to the plane of the paper, with hydrogen atoms and hydroxyl groups above or below the plane of the ring.

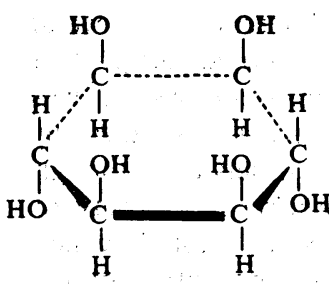

A

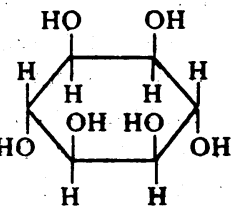

B

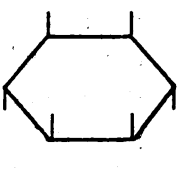

C
In 1900, Maquenne [4] devised a fractional notation whereby numerals in the numerator denote hydroxyl or other groups (not hydrogen) above the plane of the ring while numerals in the denominator denote hydroxyl or other groups (not hydrogen) below that plane. Thus the above compound received a stereochemical prefix $\frac{1,2,4,5}{3,6}$, which may be more conveniently printed as $1,2,4,5 / 3,6-$. 
Table 1. Examples of cyclitols named by different systemis

$\mathrm{P}-\mathrm{R}=$ present recommendations. $\mathrm{P}=$ Posternak [7]. FAL = Fletcher, Anderson and Lardy [5]. AG = Angyal and Gilman [6]
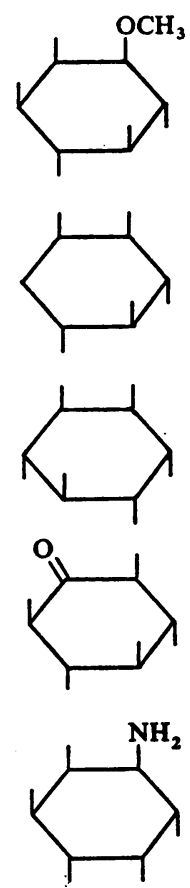

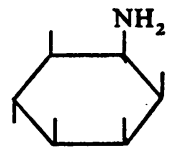

P-R: iD-1-O-Methyt-myo-inositol

P:: 3-O-Methylmyoinositol

FAL: L-1-O-Methyl-myo-inositol

AG: (1S)-1-0-Methyl-myo-inositol

Trivial

name: (-)-Bornesitol

P-R: 1L-1,2,4/3,5-Cyclohexanepentol

P: $\quad 1,2,4 / 3,5-$ Cyclohexanepentol

FAL: D-1-Deoxy-myo-inositol

AG: $\quad(1 R)$-vibo-Quercitol

Trivial

name: (-)-Viburnitol

P-R: D-chiro-Inositol

P:: (+)-Chiroinositol, 1,2,5/3,4,6-inositol

FAL: D-Inositol

AG: (1S)-Inositol, (1S)-1,2,4/3,5,6-inositol

P-R: 2,4,6/3,5-Pentahydroxycyclohexanone

P: $\quad$ Scyllomesoinosose, mesoinosose-2

FAL: myo-Inosose-2

AG: scyllo-Inosose

P-R: 1D-1-Amino-1-deoxy-neo-inositol

P: Neoinosamine-3.

FAL: L-neo-Inosamine-1

AG: (1S)-1-Amino-1-deoxy-neo-inositol

P-R: DL-2-Amino-2-deoxy-epi-inositol

P: ( $\quad$ )-2(4)-Amino-2(4)-deoxyepi-inositol

FAL: DL-epi-Inosamine-2

AG: ( $\quad($ )-2-Amino-2-deoxy-epi-inositol
Maquenne did not, however, specify exactly how the numerals were to be assigned to the individual positions, and as the chemistry of cyclitols developed, these assignments were made in different ways. Several systems of nomenclature were proposed $[5,6]$. Most notably, a logical and self-consistent system was developed (but not assembled as a set of rules) by Posternak [7], and his system was widely used, though with occasional variants, by others. The variety of names that resulted is illustrated in Table 1, which gives also the names derived by application of the Recommendations below.

It is an advantage of the Posternak system that the resulting fractional prefix describes not only the relative positions of the substituents but also the absolute configuration of a compound; no additional prefix such as $\mathrm{D}$ or $\mathrm{L}$, or $R$ or $S$, is needed to differentiate enantiomers since pairs of enantiomers receive different fractional prefixes. This very feature, however, entails serious disadvantages. The fractional prefix gives no indication whether a compound so specified is chiral or achiral, and for a pair of enantiomers gives no indication that they have the same relative configuration, i.e. that they are enantiomers. This is contrary to the practice in the rest of chemical literature, whereby enantiomers receive identical names except for a specific prefix denoting the chirality. Also, specification of racemates becomes somewhat. cumbrous by this system.

An alternative method of assigning numerals, based in part on previous practice $[5,6]$ and on proposals made by McCasland [8], was recommended by a majority of the Joint Cyclitol Nomenclature Sub-Committee ${ }^{1}$, and was adopted by the parent IUPAC and IUPAC-IUB Nomenclature Commissions in 1967 [1]. By this method, enantiomers receive identical fractional prefixes that specify relative configuration, but they also receive an additional prefix D or L, which specifies the chirality.

When the Tentative Rules were publisned in 1968 [1], it seemed advisable to set out detailed Rules for Posternak's system because it had been widely 
used in the literature up to that date. These nonpreferred Rules were, therefore, given in Part $\mathrm{C}$ of the Tentative Rules. However, this system has not been widely used during the past few years, and the 'nonpreferred' Rules are therefore omitted from these Recommendations.

The present Recommendations are essentially identical with the Tentative Rules, but they have been extensively rearranged in format for the convenience of their users.

\section{RECOMMENDATIONS $^{6}$}

\section{CYCLITOLS WITH ONLY HYDROXYL}

OR SUBSTITUTED HYDROXYL GROUUS

Inositols

Configurational prefixes

I-1.1. The 1,2,3,4,5,6-cyclohexanehexols are termed generically 'inositols'. Individual inositols are differentiated by use of an italicized prefix. and hyphen, as follows, the locants (positional numbers) being assigned according to criteria (ii) and (vi) of Recommendation I-4.

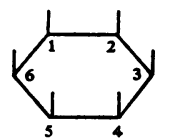

$$
\begin{aligned}
& \text { cis-Inositol } \\
& (1,2,3,4,5,6 / 0-)^{7,8} \\
& \text { allo-Inositol } \\
& (1,2,3,4 / 5,6-) \\
& \text { myo-Inositol } \\
& \text { (1,2,3,5/4,6-) } \\
& \text { (formerlỳ meso-inositol) } \\
& (1,2,4,5 / 3,6-) \\
& \text { neo-Inositol } \\
& (1,2,3 / 4,5,6-)
\end{aligned}
$$$$
\text { (1) }
$$$$
\int_{6}{ }^{2}{ }_{3}^{2} \quad \text { muco-Inositol }
$$

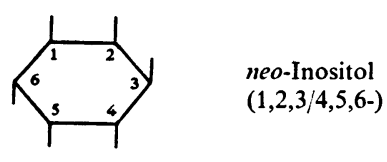

${ }^{6} \mathrm{I}$ (for Inositol) is attached to the Recommendation numbers as a general identifying prefix.

${ }^{7}$ Preferred to 'all-cis' in this and similar cases. The zero is inserted for clarity.

8 Throughout the examples, a simple vertical stroke standing alone signifies a bond to a hydroxyl group.

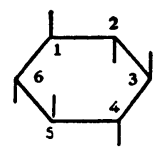

scyllo-Inositol

$(1,3,5 / 2,4,6-)$

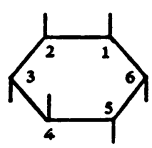

1D-chiro-Inositol ${ }^{9}$

$(1,2,4 / 3,5,6)$

[formerly $\mathrm{D}$ - or

(t)-inositol]

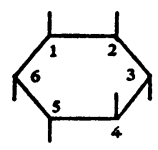

1L-chiro-Inositol ${ }^{9}$
$(1,2,4 / 3,5,6)$
[formerly $L$ - or
(-)-inositol]

I-1.2. The numberings of formulas (1)-(9) are retained for derivatives of the inositols. Within this framework, criteria (iv) and (v) of Recommendation I-4 are used to decide between alternatives. These arise because (a) in several of the parent inositols [(1), (5), (6), (7), (8) and (9)] there are two or more fully equivalent starting points for numbering that may not be equivalent in the derivatives, and (b) criterion (vi) of Recommendation I-4 does not apply to chiral derivatives of the meso-inositols $[(1)-(7)]$. The application of criteria (iv) and (v) to a pair of enantiomers gives each pair of mirror-related positions the same number. Typically one enantiomer will be numbered clockwise, the other counterclockwise.

\section{Other Cyclitols}

\section{Trivial Names}

I-2. The trivial name (+)-quercitol is permitted for $1 \mathrm{~L}-1,3,4 / 2,5$-cyclohexanepentol (for derivation of this name see below). The generic name 'quercitols' is abandoned.

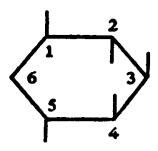

$$
\text { (+)-Quercitol }
$$

\section{Description of Structure}

I-3. The structure of cyclitols other than the inositols are described by use of the IUPAC 1971 Rules for the Nomenclature of Organic Chemistry, Section C [9], with the proviso that cyclitols are named as substituted cycloalkanepolyols even when some or all of the hydroxyl groups are substituted.

\footnotetext{
I-10.

${ }^{9}$ For absolute configurational prefixes, see Recommendation
}

Vol. 153 


\section{Assignment of Locants (Positional Numbers)}

I-4. Locants (positional numbers) are assigned to the carbon atoms of the ring, and thus the direction of numbering is described, with reference to the steric relations and nature of the substituents attached to the ring. The substituents lying above the plane of the ring constitute a set, and those lying below the plane another set. Lowest locants are related to one set of the substituents according to the following criteria, which are applied successively until a decision is reached:

(i) to the substituents considered as a numerical series, without regard to configuration;

(ii) if one set of substituents is more numerous than the other, to the more numerous;

(iii) if the sets are equally numerous and one of. them can be denoted by lower numbers, to that set;

(iv) to substituents other than unmodified hydroxyl groups;

(v) to the substituent first in alphabetical order (Rule C-16.3 in [9]);

(vi) for meso-compounds only - to those positions that lead to an $\mathrm{L}$ rather than a $\mathrm{D}$ designation when Recommendation I-10 is applied to the lowestnumbered asymmetric carbon atom.

Notes

a) 'Lowest numbers' are those that, when considered as a single ascending series, contain the lower number at the first point of difference, e.g. 1,2,3,6 is lower than $1,2,4,5$.

b) Criterion (vi) is needed only for compounds with meso-configuration, being required only for problems involving prochirality [10]. It can be simply applied by noting that it causes numbering to be clockwise when the formula is oriented so that the substituent on the lowest-numbered asymmetric carbon atom of the ring projects upwards.

c) When two or more positions are fully equivalent it is immaterial which is chosen as the starting point.

\section{Relative Configuration}

I-5.1. The relative configurations at ring positions of a cyclitol, other than an inositol or a derivative thereof, are described by means of a fraction. The numerator of the fraction consists of the locants (assigned as described above) of the set of substituents that lies above or below the plane of the ring, these numbers being arranged in ascending order and separated by commas. The denominator contains the locants of the other set. Conventionally, the set of locants containing the lowest numbers is cited as numerator.
I-5.2. When only hydroxyl or substituted hydroxyl groups are involved, the fraction also serves as a list of locants. Its position in the name is that usually assigned to the list of locants (see examples).

\section{Note}

The fraction may be written with a horizontal or a sloping division line e.g. $\frac{1,2,4}{3,5,6}$ or $1,2,4 / 3,5,6-$.

Examples $^{10}$ (showing also which criterion of Recommendation I-4 was applied):

(i)

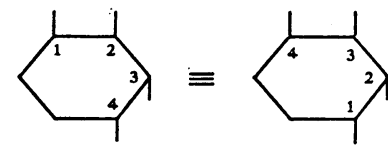

$1 \mathrm{~L}-1,2 / 3,4-$

Cyclohexanetetrol $^{11}$ (11)

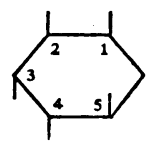

1D-1,2,5/3,4-Cyclohexanepentol (12)

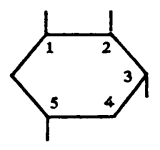

1L-1,2/3,5-Cyclohexanetetrol

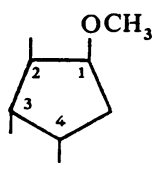

1D-1-O-Methyl-1,2/3,4cyclopentanetetrol

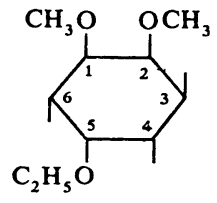

1L-5- $O$-Ethyl-1,2-di- $O$-methylneo-inositol (Note: numbers $1,2,5$ for substituents are lower than $2,3,5$ or $2,4,5$ or $2,5,6$ )

$(i v, v)$

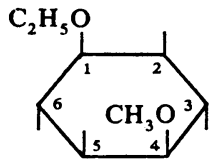

1L-1-O-Ethyl-4- $O$-methylmuco-inositol

\footnotetext{
${ }^{10}$ As exceptions to general nomenclature, but in accord with carbohydrate nomenclature, ethers and esters of cyclitols may be named either by using prefixes such as 1-O-methyl, 1-O-acetyl, etc. or by adding 1-methyl ether, 1 -acetate, etc., after the name of the polyol. For simplicity, only the former alternative is used in these examples.

${ }^{11}$ For allocation of $D$ and $L$ to these and other enantiomers in the examples, see Recommendation I-10.
} 
$(i, v i)$<smiles>CC1CCC(C)C(C)CC1</smiles>

\section{1,2,3,4,5/0-Cycloheptanepentol}

(ii, vi)<smiles>CO[C@]12CC3CCC(C3)C1C2</smiles>

2-O-Methyl-mỵo-inositol

CYCLITOLS WITH GROUPS OTHER THAN HYDROXYL OR SUBSTITUTED HYDROXYL

\section{Inositol Derivatives}

\section{Trivial Names}

I-6. $x$-Amino- $x$-deoxyinositols are termed generically 'inosamines'; individual compounds of this group are named according to Recommendation I-7.1.

\section{Systematic Nomenclature}

1-7.1. If one, two or three hydroxyl groups of an inositol are replaced by other univalent substituents with retention of configuration, and if, according to the IUPAC 1971 Rules for the Nomenclature of Organic Chemistry, Section C. [9], these substituents need not be named as suffixes, the compound is regarded as a substituted inositol and the 'deoxy' nomenclature is used. The configurational prefix and the numbering of the parent inositol are retained. (For cyclitols, the most important part of the order of decreasing priority for citation as suffix is: $\mathrm{COOH}$ and modified $\mathrm{COOH},=\mathrm{O}, \mathrm{OH}, \mathrm{SH}, \mathrm{NH}_{2}$.) When this leaves alternatives criteria (iv) to (vi) of Recommendation I-4 are applied.

If a substituent that must be named as a suffix is present, the compound is named according to Recommendations I-8 and I-9.

I-7.2. Inositol derivatives in which one carbon atom carries a substituent additional to a hydroxyl are named as substituted inositols, provided that the substituent does not rank above hydroxyl for citation as suffix. (If it does, Recommendation I-9.2 applies.) For the disubstituted position in such compounds the configurational prefix refers to the hydroxyl group.

Examples:

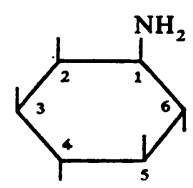

1D-1-Amino-1-deoxy-myo-inositol

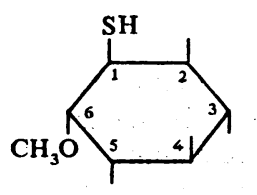

1L-1-Deoxy-1-mercapto-6-O-methylchiro-inositol ${ }^{12}$
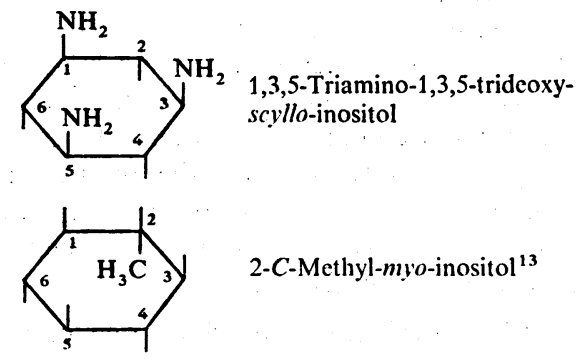

2-C-Methyl-myo-inositol ${ }^{13}$

\section{Other Cyclitols}

\section{Trivial Names}

I-8. The 2,3,4,5,6-pentahydroxycyclohexanones are termed generically 'inososes'; the individual compounds are named according to Recommendation I-9. The trivial name (-)-quinic or L-quinic acid is preferred for the following compound (see the last example to Recommendation I-9):

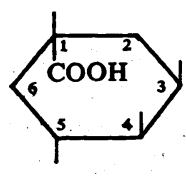

(-)-Quinic acid; L-Quinic acid; 1L-1(OH),3,4/5-Tetrahydroxycyclohexanecarboxylic acid

\section{Systematic Nomenclature}

I-9.1. Cyclitols containing substituents other than hydroxyl or modified hydroxyl (excepting inositols covered by Recommendation I-7) are named and numbered according to the above-mentioned IUPAC Rules, with the proviso that $O$-substituents are named as such (see Recommendation I-3). Substituted hydroxyl groups are not named as alkoxy, aryloxy or acyloxy groups. When these rules leave alternatives available, the criteria (ii) to (vi) of Recommendation I-4 are applied. Relative configuration is indicated as prescribed in Recommendation I-5.1. Except when it is serving as a locant set, the fraction describing the configuration is placed in parentheses, and it becomes the first element in the name, except for the configurational prefix (Recommendation I-10).

\footnotetext{
12 IUPAC Rule C-502 is compatible with a name 1L-6-Omethyl-1-thio-chiro-inositol but that name does not accord with the instruction in Recommendation I-7.1 to use the 'deoxy' nomenclature.

${ }^{13}$ The prefix $C$ - is added to denote substitutions on carbon in accordance with carbohydrate nomenclature [11].
}

Vol. 153 


\section{Notes}

The IUPAC Rule C-10 [9] provides that one type of group be chosen as suffix, named as suffix, and given the lowest possible number(s), the remaining types prefixes. For choice of suffix, see the penultimate (parenthetical) sentence in Recommendation I-7.1.

When there are substituents other than hydroxyl groups, it is usually impracticable to use the fraction describing the relative configuration as a list of locants. In stipulating that, in such cases, the fraction be placed in front of the complete name of the compound (including $O$-substituents), the present Recommendations differ from the Tentative Rules.

I-9.2. Cyclitol derivatives in which one carbon atom carries a substituent additional to hydroxyl are named (a) as substituted cycloalkanepolyols or (b) as hydroxy derivatives, according as a substituent (a) does not or (b) does rank above hydroxyl for citation as suffix. When the Recommendation leaves alternatives available, the criteria (ii) to (vi) of Recommendation I-4 are applied. For the disubstituted positions in such compounds, the fractional prefix refers to the hydroxyl group and this may be specified for clarity where necessary.

\section{Note}

Replacement of the hydrogen of amino, mercapto or hydroxyl groups by other atoms or groups does not change the numbering of cyclitol derivatives except when it affects criterion (iv) or (v) of Recommendation I-4. However, the IUPAC Rules [9] require that the ring carbon atom carrying as a substituent a trisubstituted ammonio $\left(\mathrm{R}_{3} \mathrm{~N}^{+}\right)$, acid, oxo, cyano or acyl group, or a derivative thereof, receive the locant 1 ; such cases will be relatively rare in cyclitol chemistry. A convenient alternative for 'onium' salts is to use the terminology exemplified by methiodide, hydrochloride, sulfate, etc.

Examples:

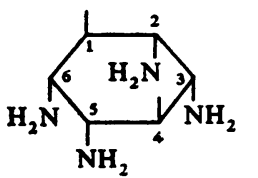

$(1,4 / 2,3,5,6)-2,3,5,6$-Tetraamino1,4-cyclohexanediol

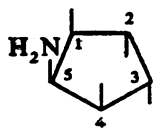

(1,4,5/2,3)-5-Amino-1,2,3,4cyclopentanetetrol

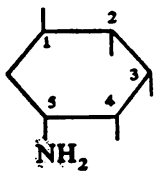

1L-(1/2,3,4,5)-5-Amino-1,2,3,4cyclohexanetetrol

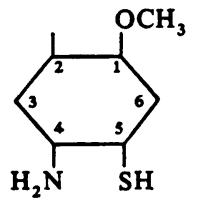

1D-(1,2/4,5)-4-Amino-5-mercapto-

1-O-methyl-1,2-cyclohexanediol

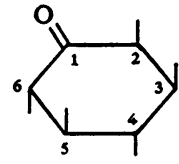

2L-2,3,5/4,6-Pentahydroxycyclohexanone<smiles>CC1CC2CC2(C(=O)O)C1</smiles>

$1 \mathrm{~L}-(1,5 / 2,3,4)-2,3,4,5$ -

Tetrahydroxycyclopentanecarboxylic acid

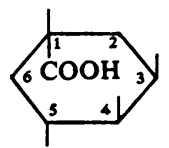

$1 \mathrm{~L}-1(\mathrm{OH}), 3,4 / 5$ -

Tetrahydroxycyclohexanecarboxylic acid ( $\mathrm{L}$-quinic acid) (this numbering is opposite to former usage)

\section{ABSOLUTE CONFIGURATION}

I-10. The absolute configuration of a cyclitol is specified by making a vertical Fischer-Tollens type of projection of the structure, with C-1 at the top and with $\mathrm{C}-2$ and $\mathrm{C}-3$ on the front edge of the ring. The configuration is then designated as $\mathrm{D}$ if the hydroxyl group at the lowest-numbered chiral center (or other substituent if no hydroxyl group is present there) projects to the right, and as $\mathrm{L}$ if it projects to the left (cf. Fig. 1). The prefix D or L, followed by a hyphen, is written before the name of the compound and may be preceded by the locant of the defining center. Racemic compounds are designated by the prefix DL.

\section{Notes}

a) The mere absence of a prefix $D, L$, or DL indicates that the compound has a meso-configuration; thus, the prefix D, L, or DL should not be omitted.

b) A simple way of applying this Recommendation is as follows: when the formula is drawn in such a way that the substituent on the lowest-numbered asymmetric carbon atom is above the plane of the
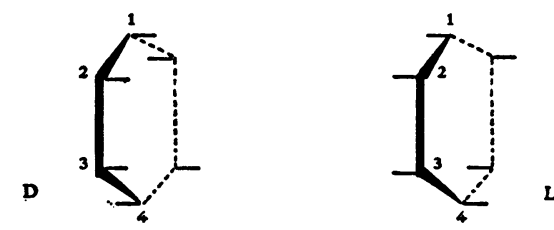

Fig. 1 
ring, and the numbering is clockwise, the compound is $\mathrm{L}$; if anti-clockwise, it is D [see examples (8) and (9)].

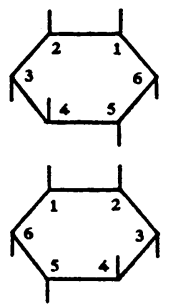

1D-chiro-Inositol

1L-chiro-Inositol

c) In a great majority of cases the lowest-numbered chiral center is position 1, so that it would be reasonable that $\mathrm{D}$ or $\mathrm{L}$ should be preceded by the locant of the defining center only when it is not 1 . However, according to another nomenclature system for cyclitols [5] and also for the related carbohydrate field, the symbols $D$ and $L$ are assigned to the highestnumbered chiral center, which sometimes gives symbols different from those assigned according to Recommendation I-10. It is, therefore, recommended that the numeral 1 be included (as in these Recommendations).

d) Small Roman capital letters should be used in print for $\mathrm{D}$ and $\mathrm{L}$. For compounds containing cyclitols and protein or carbohydrate residues, $D_{c}$ and $\dot{L}_{c}$ (c for cyclitol) may be used alongside $D_{s}, L_{s}, D_{g}$, and $L_{B}[12]$. Cyclitol nomenclature may also be combined with the use of the sequence rule $[2,3]$ or the stereospecific numbering (sn) system [13], where necessary.

e) When many hydroxyl groups are replaced by other substituents, it may be simpler to use the sequence rule [2,3]. Sequence-rule examples are given in Table 1, also in ref. [2,3].

Examples:

Many examples of chiral compounds are named in the preceding examples [e.g. (11)-(16); (26)-(29)]. The following are additional.
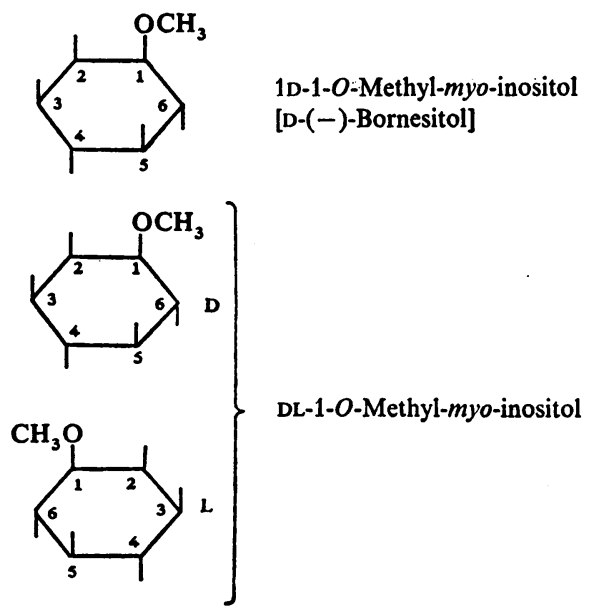

Vol. 153
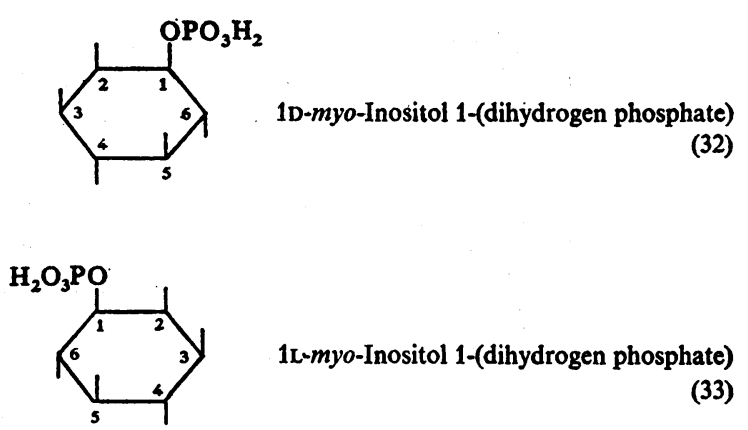

1L-myo-Inositol 1-(dihydrogen phosphate)

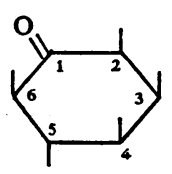

2L-2,3,4,6/5-Pentahydroxycyclohexanone

Acknowledgements. The formulae are reproduced directly from Pure Appl. Chem. 37, 285-297 (1974) by kind permission of I.U.P.A.C. and Messrs Butterworths, London.

\section{REFERENCES}

1. IUPAC Commission on the Nomenclature of Organic Chemistry and IUPAC-IUB Commission on Biochemical Nomenclature (1968) IUPAC Inf. Buill. 32, 51; see also Eur. J. Biochem. 5, 1 (1968); Arch. Biochem. Biophys. 128, 269 (1968); J. Biol. Chem. 243, 5809 (1968); Biochem. J. 112, 17 (1969); Biochim. Biophys. Acta, 165, 1 (1968); German langnage version in Hoppe-Seyler's Z. Physiol. Chem. 350, 523 (1969); French-langnage version in Bull. Soc. Chim. Biol. 51, 3(1969).

2. Cahn, R. S., Ingold, C. \& Prelog, V. (1966). Angew. Chem. 78, 413; Angew. Chem. Int. Ed. 5, 385

3. International Union of Pure and Applied Chemistry (1970) J. Org. Chem. 35, 2849; see also Eur. J. Biochem. 18, 151 (1971).

4. Maquenne, L. (1900) Les sucres et leurs principaux derivés, Ganthiers-Villars, Paris; see also Carrè, G. \& Naud, C. (1900) Paris.

5. Fletcher, H. G., Jr, Anderson, L. \& Lardy, H: A. (1951) J. Org. Chem. 16, 1238.

6. Angyal, S. J. \& Gilham, P. T. (1957) J. Chem. Soc. (Loind.) 3691.

7. Posternak, T. (1965) The Cyclitols, Hermann, Paris.

8. McCasland, G. E. (1965) Adv. Carbohydr. Chem. 20, 11.

9. International Union of Pure and Applied Chemistry (1971) Definitive Rules for Nomenclature of Organic Chemistry, 2nd edn, Section C, Butterworths, London.

10. Hanson, K: R. (1966) J. Am. Chem. Soc. 88, 2731.

11. IUPAC Commission on the Nomenclature of Organic Chemistry and IUPAC-IUB Commission on Biochemical Nomenclature (1970) IUPAC Inf. Bull. Append. 7; see also Eur. J. Biochem. 21, 455 (1971).

12. International Union of Pure and Applied Chemistry (1960) J. Am. Chem. Soc. 82, 5575.

13. IUPAC-IUB Commission on Biochemical Nomenclature (1967) Eur. J. Biochem. 2, 127 (1967). 
Acknowledgement. We are indebted to the Editors and Publishers (Springer-Verlag) of the European Journal of Biochemistry for permission to reproduce these Recommendations photographically.

All Tentative Rules and Proposals of the IUPAC-IUB Commission of Biochemical Nomenclature $(\mathrm{CBN})$ are available from Waldo E. Cohn, Director, NRC Office of Biochemical Nomenclature, Oak Ridge National Laboratory, Box Y, Oak Ridge, Tenn. 37830, U.S.A.

1. Abbreviations and Symbols for Chemical Names of Special Interest in Biological Chemistry [see Biochem. J. (1966) 101, 1-7 (extended by items 6, 11 and 15 below)].

2. Nomenclature of Vitamins, Coenzymes and Related Compounds: Trivial Names of Miscellaneous Compounds of Importance in Biochemistry. Nomenclature of Quinones with Isoprenoid Side Chains, Nomenclature and Symbols for Folic Acid and Related Compounds, Nomenclature of Corrinoids. Tentative Rules [see Biochem. J. (1967) 102, 15-22 (but see items 10, 22, 23 and 24 below)].

3. Abbreviated Designation of Amino Acid Derivatives and Peptides [see Biochem.J. (1967) 102, 23-27 (superseded by item 15 below)].

4. Rules for Naming Synthetic Modifications of Natural Peptides [see Biochem. $J$. (1967) 104, 17-19 (for amendments see Biochem. J. (1973) 135, 9)].

5. The Nomenclature of Lipids. A Document for Discussion [see Biochem. J. (1967) 105, 897-902 (for corrections see correction slip in Biochem. J. (1970) 116, issue no. 5)].

6. Abbreviated Nomenclature of Synthetic Polypeptides (Polymerized Amino Acids).

Tentative Rules [see Biochem. J. (1968) 106, 577-579 (superseded by item 18 below)].

7. The Nomenclature of Cyclitols. Tentative Rules [see Biochem. J. (1969) 112, 17-28 (extended by this document)].

8. A One-Letter Notation for Amino Acid Sequences. Tentative Rules [see Biochem. J. (1969) 113, 1-4].

9. Revised Tentative Rules for Nomenclature of Steroids [see Biochem. J. (1969) 113, 5-28 (for amendments see item 16 below)].

10. Nomenclature for Vitamins $\mathbf{B}_{6}$ and Related Compounds. Tentative Rules [see Biochem. J. (1970) 119, 1-4 (replaces M-7 of item 2 above; superseded by item 20 below)].

11. Abbreviations and Symbols for Nucleic Acids, Polynucleotides and their Constituents. Recommendations [see Biochem. J. (1970) 120, 449-454 (replaces section 5 of item 1 above)].

12. Abbreviations and Symbols for the Description of the Conformation of Polypeptide Chains. Tentative Rules [see Biochem. J. (1971) 121, 577-585].

13. Tentative Rules for Carbohydrate Nomenclature: Part 1 [see Biochem. J. (1971) 125, 673-695].

14. The Nomenclature of Multiple Forms of Enzymes. Recommendations [see Biochem. J. (1972) 126, 769-771].

15. Symbols for Amino Acid Derivatives and Peptides. Recommendations [see Biochem.J. (1972) 126, 773-780 (supersedes item 3 above; for corrections see Biochem.J. (1973) 135, 9)].

16. Amendments to Rules for Nomenclature of Steroids [see Biochem. J. (1972) 127, 613-617 (amendments to item 9 above)].

17. Tentative Rules for the Nomenclature of Carotenoids [see Biochem. J. (1972) 127, 741-752].

18. Abbreviated Nomenclature of Synthetic Polypeptides (Polymerized Amino Acids). Revised Recommendations [see Biochem. J. (1972) 127, 753-756 (supersedes item 6 above)].

19. Nomenclature of Iron-Sulphur Proteins. Recommendations [see Biochem. J. (1973) 135, 5-7].

20. Nomenclature for Vitamins B-6 and Related Compounds. Recommendations [see Biochem. J. (1974) 137, 417-421 (supersedes item 10 above)].

21. Recommendations for the Nomenclature of Human Immunoglobulins [see Biochem. J. (1975) 145, 21-23]. 
22. The Nomenclature of Corrinoids. Recommendations [see Biochem. J. (1975) 147, 1-10 (supersedes section in item 2 above)].

23. Nomenclature of Tocopherols and Related Compounds. Recommendations [see Biochem. J. (1975) 147, 11-14 (replaces M-3 of item 2 above)].

24. Nomenclature of Quinones with Isoprenoid Side Chains. Recommendations [see Biochem. J. (1975) 147, 15-21 (supersedes section in item 2 above)].

25. Nomenclature of $\alpha$-Amino Acids. Recommendations [see Biochem. J. (1975) 149, 1-16].

26. The Nomenclature of Peptide Hormones. Recommendations [see Biochem. J. (1975) 151, 1-4].

27. Nomenclature of Carotenoids. Recommendations [see Biochem. J. (1975) 151, 5-7 (amendments to item 17 above)].

28. Nomenclature of Cyclitols. Recommendations (this document).

A document, OBN-5, describing the (American) NRC Office of Biochemical Nomenclature, and listing other rules affecting biochemical nomenclature, is available from its Director, Dr. Waldo E. Cohn [see also J. Chem. Doc. (1967) 7, 72-73; (1969) 9, 235-241]. 